\title{
Gestação na adolescência precoce e tardia - há diferença nos riscos obstétricos?
}

\author{
Precocious and late pregnancy in adolescents - \\ is there a difference comparing the obstetric risks?
}

\begin{abstract}
Maria de Lourdes Caltabiano Magalhães ${ }^{1}$, Felipe Magalhães Furtado ${ }^{2}$, Marcelo Bezerra Nogueira ${ }^{3}$, Francisco Herlânio Costa Carvalho ${ }^{4}$, Francisco Manuelito Lima de Almeida ${ }^{4}$, Rosiane Mattar ${ }^{5}$, Luiz Camano ${ }^{5}$
\end{abstract}

\section{RESUMO}

Objetivos: descrever aspectos da assistência e resultados obstétricos da gravidez em adolescentes atendidas em um centro de atendimento terciário do Ceará e comparar os resultados maternos e perinatais entre adolescentes precoces e tardias. Métodos: em estudo transversal, analítico, avaliaram-se 2.058 casos, sendo 322 (15,6\%) de adolescentes precoces e 1.736 $(84,4 \%)$ tardias, atendidas no ano de 2000 . Foram analisados as intercorrências clínicas no pré-natal, tipo de parto, indicações de cesárea, idade gestacional no parto, peso do recém-nascido ao nascimento, adequação do peso à idade gestacional, índices de Apgar no primeiro e quinto minuto de vida, presença de malformações e óbito neonatal. Utilizaram-se o teste exato de Fisher e o $\chi^{2}$ na comparação entre os dois grupos. Calculou-se também a razão de prevalência. Resultados: do total de partos ocorridos no período, 25,9\% eram de adolescentes e a média de idade destas foi de 17,2 anos. Constatouse que $88 \%$ freqüentaram o pré-natal, sendo $60 \%$ com número insuficiente de consultas. As intercorrências clínicas mais freqüentes foram a pré-eclâmpsia $(14,7 \%)$, a anemia $(12,9 \%)$ e a infecção do trato urinário $(6,4 \%)$, sem diferença de frequiência entre os grupos. Ocorreram $31,3 \%$ de nascimentos por cesárea, sendo a pré-eclâmpsia a principal indicação nas duas faixas etárias (25 e 23\%, respectivamente). A freqüência de Apgar menor que 7 no primeiro minuto foi de $19,9 \%$ no grupo das adolescentes precoces e $14,2 \%$ entre as tardias $\left(x^{2}=6,96, p=0,008\right)$. Não houve diferença quanto à frequiência de prematuridade $(20,2$ vs 16,1\%), recém-nascido pequeno para idade gestacional (12,4 vs 10,4\%), baixos escores de Apgar de quinto minuto $(5,3$ vs 3,3\%), malformações congênitas $(3,1$ vs $2,7 \%)$ e morte neonatal (1,6 vs 3,1\%). Conclusões: as gestantes adolescentes precoces e tardias apresentaram evolução da gestação e desempenho obstétrico semelhantes, exceto pela diferença nos escores de Apgar no primeiro minuto.

PALAVRAS-CHAVE: Gravidez na adolescência; Pré-eclâmpsia; Assistência perinatal; Resultado da gravidez

\section{ABSTRACT}

Purpose: to describe the obstetric outcomes in pregnant adolescents at a tertiary hospital and to compare the maternal and labor outcomes between precocious and late adolescents. Methods: in a transversal analytical study, 2058 cases were evaluated, considering $322(15.65 \%)$ from the precocious group and $1736(84.35 \%)$ from the late group that delivered at the "Maternidade Escola Assis Chateaubriand/UFC" from January 1, 2000 to December 31, 2000. The clinical complications in the prenatal period, kind of delivery, indications for cesarean section, birth gestational age at birth, birth weight, comparison of birth weight and gestational age, Apgar score at the first and fifth minute, presence of malformations, and neonatal death were analyzed. The exact Fisher and the $\chi^{2}$ tests were used to compare both groups. The prevalence ratio was calculated. Results: from of total of deliveries, $25.95 \%$ belonged to adolescents. The average age was 17.19 years. Prenatal visits were made by $88 \%$ of the patients, but $60 \%$ had an insufficient number of visits. The most frequent clinical situations were preeclampsia (14.72\%), anemia (12.97\%) and urinary tract infections (6.37\%), with no statistical difference

\footnotetext{
Maternidade-Escola Assis Chateaubriand - Universidade Federal do Ceará - UFCE - Fortaleza (CE), Brasil.

1 Médica da Maternidade-Escola Assis Chateaubriand - Universidade Federal do Ceará UFCE - Fortaleza (CE), Brasil.

2 Acadêmico de Medicina da Universidade Federal do Ceará - UFCE - Fortaleza (CE), Brasil.

Professor do Departamento de Matemática e Estatística da Universidade Federal do Ceará - UFCE - Fortaleza (CE), Brasil.

Professor do Departamento de Saúde Materno-Infantil da Universidade Federal do Ceará - UFCE - Fortaleza (CE), Brasil.

5 Professor do Departamento de Obstetricia da UNIFESP - Escola Paulista de Medicina - São Paulo (SP), Brasil. Correspondência:

Maria de Lourdes Caltabiano Magalhães

Rua República do Líbano $\mathrm{n}^{\circ}$ 630, apt. 702 - Bairro: Meireles - 60160-140 - Fortaleza - CE - Telefone: (85) 3224-3342 - Fax: (85) 32615676 - e-mail: mariadelourdes@uol.com.br

Recebido em: 31/10/2005

Aceito com modificações em: 12/07/2006
}

Rev Bras Ginecol Obstet. 2006; 28(8): 446-52 
between the groups. Thirty-one and three percent of the births were by cesarean section, preeclampsia being the main indication in the two age groups ( 25 and 23\%, respectively). The frequency of an Apgar score less than 7 at the first minute was $19,9 \%$ in the precocious adolescent group and $14,2 \%$ in the late adolescent group $\left(x^{2}=6,96, p=0.008\right)$. There was no statistical difference regarding prematurity rate (20.2 vs 16.1\%), low-birth weight infants (12.4 vs 10.4\%), low Apgar score at the fifth minute (5.3 vs 3.3\%), congenital malformations (3.1 vs 2.7\%), and neonatal death (5.3 vs 3.3\%). Conclusions: the precocious and late pregnant adolescents presented similar pregnancy evolution and obstetric outcomes, except for the differences of the first minute Apgar scores.

KEYWORDS: Pregnancy in adolescence; Pre-eclampsia; Perinatal care; Pregnancy outcome

\section{Introdução}

As adolescentes, pelas próprias características associadas à faixa etária, ainda não são capazes de avaliar, e principalmente assumir, o ônus da vida sexual ativa. Estima-se que cerca de 15-20\% de todos os nascimentos ocorram em mulheres adolescentes e, embora a freqüência de partos em adolescentes esteja em declínio nos países desenvolvidos, há somente modesto declínio ou até ascensão nestas taxas nos países em desenvolvimento. Portanto, a gestação na adolescência persiste como importante problema de saúde pública nestes países ${ }^{1}$.

A gravidez na adolescência tem sido associada a uma freqüência aumentada de resultados obstétricos adversos, tais como baixo peso ao nascer, parto prematuro, morte materna e perinatal, préeclâmpsia e parto cirúrgico. Não se sabe se estas complicações são relacionadas a fatores biológicos ou sócio-econômicos ${ }^{2,3}$. No entanto, outros relatos sugerem que gestantes adolescentes apresentam resultados obstétricos favoráveis e não devem ser consideradas de alto risco ${ }^{4,5}$.

Todavia, as pesquisas nesta área apresentam muitas limitações metodológicas, como por exemplo: pequeno tamanho da amostragem, ausência de uniformidade nas definições das complicações avaliadas e falta de controle sobre fatores potencialmente capazes de interferir nos resultados perinatais que não apenas a idade materna ${ }^{6}$. Há, especialmente, pouca informação acerca da dúvida se adolescentes mais jovens (precoces) teriam prognóstico obstétrico semelhante ou não ao das adolescentes mais velhas (tardias). Estudos sobre grávidas adolescentes, tipicamente, limitam sua população à idade de 15 a 19 anos, enquanto informações sobre mulheres mais jovens aparecem somente em estatísticas agregadas. Os poucos estudos que incluíram adolescentes mais jovens não possuem conclusões homogêneas em relação aos riscos associados à concepção nessa população. Isto pode refletir inconsis- tência na definição do limite para considerar a adolescente precoce ${ }^{7}$.

O objetivo do presente estudo foi descrever os resultados obstétricos nas adolescentes grávidas em centro de atendimento terciário e comparar os resultados maternos e perinatais entre adolescentes precoces e tardias.

\section{Métodos}

Trata-se de estudo transversal, analítico. Foi desenvolvido na Maternidade-Escola Assis Chateaubriand/Universidade Federal do Ceará (MEAC/UFC) no período de 01 de janeiro de 2000 a 31 de dezembro de 2000.

Os dados sobre as gestantes e seus recémnascidos (RN) foram adquiridos de forma retrospectiva. O protocolo de pesquisa foi analisado e aprovado pelos Comitês de Ética em Pesquisa das Universidades Federais do Ceará e de São Paulo e segue os termos preconizados pelo Conselho Nacional de Saúde (portaria 196/96).

Foram incluidas 2.058 parturientes que apresentavam informações completas no prontuário médico no período do estudo e que apresentavam no máximo 19 anos e 364 dias de idade. Do total de partos no período considerado, 25,9\% ocorreram em adolescentes.

As variáveis analisadas foram: intercorrências clínicas no pré-natal, tipo de parto (vaginal, fórcipe ou cesariana), indicações da cesariana (pré-eclâmpsia, distocia funcional, desproporção céfalo-pélvica, sofrimento fetal, cesárea de repetição), idade gestacional no momento do parto (freqüência de prematuridade), adequação do peso no nascimento (freqüência de recém-nascidos pequenos para idade gestacional), índices de Apgar no primeiro e quinto minutos de vida, presença de malformações congênitas e óbito neonatal. Como fatores possivelmente influenciadores dos resultados obstétricos foram avaliados: acesso e número de consultas ao pré-natal e uso de fumo, álcool ou drogas ilícitas. 
A idade materna foi definida como idade da mãe em anos completos na época do parto e foi categorizada em 2 grupos: pacientes com idade inferior a 16 anos - adolescentes precoces - e pacientes com idade entre 16 e 19 anos e 364 dias - adolescentes tardias ${ }^{7,8}$. Foram consideradas usuárias de fumo, álcool ou drogas ilícitas quando qualquer quantidade dessas substâncias foi usada durante a gestação. A idade gestacional foi definida como o número de semanas completas do primeiro dia do último período menstrual até o dia do parto. Foi considerado parto prematuro quando ocorria entre 20 semanas completas e 36 semanas e 6 dias de evolução. O tipo de parto foi considerado cesárea, fórcipe ou vaginal. O peso do recém-nascido foi verificado no momento do nascimento e expresso em gramas. A presença de $\mathrm{RN}$ pequenos para a idade gestacional foi definida quando o peso encontrava-se menor que o percentil 10 para a idade gestacional calculada pelo método de Capurro. O escore de Apgar foi classificado em duas categorias: inferior a 7 e superior ou igual a 7 e avaliado no $1^{\circ}$ e $5^{\circ}$ minuto de vida. A presença de malformações foi definida em exame físico logo após o nascimento ou durante o período neonatal precoce. O óbito neonatal foi considerado quando ocorria até o final do período de 28 dias de vida. A pré-eclâmpsia foi diagnosticada quando a pressão arterial encontrava-se superior ou igual a 140 × $90 \mathrm{mmHg}$ e a proteinúria no Labistix de $1+$ em dois episódios ou $2+$ em um único episódio. A infecção do trato urinário foi definida quando a urocultura encontrava-se positiva com contagem de mais de 100.000 unidades formadores de colônias ou sumário de urina mostrando mais de 5 leucócitos por campo ou nitrito positivo em pacientes sintomáticas. A anemia foi verificada quando a hemoglobina era inferior a $11 \mathrm{~g} / \mathrm{dL}$ em pelo menos uma oportunidade no pré-natal. O pré-natal foi considerado adequado quando o número de consultas foi superior ou igual a 6 e inadequado quando inferior a 6 .

A amostra foi constituída por $322(15,7 \%)$ adolescentes precoces e $1.736(84,3 \%)$ tardias. O total de recém-nascidos foi de 2.083 , pois ocorreram 25 casos de gemelaridade, sendo 1 caso entre as adolescentes precoces $(0,3 \%)$ e $24(1,4 \%)$ entre as tardias.

Utilizaram-se o teste exato de Fisher e o $\chi^{2}$ na comparação entre os dois grupos. Adotou-se o nivel de significância de 0,05 (alfa de 5\%) e níveis inferiores a esse valor foram considerados significantes. Calculou-se também a razão de prevalência.

\section{Resultados}

A idade das parturientes variou de 11 a 19 anos. A média de idade foi de 17,2 ( $\pm 1,5$ anos) e a idade mais freqüente foi 19 anos. Houve aumento linear com a progressão da idade materna: 11 anos (1 caso: 4,2\%), 12 anos (4), 13 anos (19), 14 anos (87), 15 anos (211), 16 anos (323), 17 anos (418), 18 anos (476) e 19 anos (519 casos: 25,2\%).

Não havia informação sobre o estado conjugal em 213 prontuários. Um total de 65,6\% (1.190) das pacientes se declararam casadas ou com união consensual e $35,5 \%$ (655) solteiras. Um total de $58,2 \%(163 / 280)$ das adolescentes precoces e $65,6 \%$ das tardias $(1.027 / 1.565)$ mantinham relacionamentos estáveis $\left(\chi^{2}=5,69 ; \mathrm{p}=0,017\right)$.

A maioria das pacientes era primigesta $(1.500=73,1 \%)$. Entre as adolescentes precoces, $93,2 \%(300 / 322)$ eram primiparas, enquanto $69,3 \%$ das tardias $(1.200 / 1.631)$ encontravam-se no primeiro parto $\left(\chi^{2}=78,9 ; \mathrm{p}<0,0001\right)$. O nível de escolaridade (tempo de estudo em anos) também foi maior no grupo das adolescentes tardias: $32,2 \%$ (482/1.495) estudaram por tempo maior ou igual a oito anos. No grupo das precoces 15,4\% (41/267) atingiram esse tempo de estudo.

Das 322 adolescentes precoces, $11(3,4 \%)$ eram tabagistas, isto é, fumavam mais de 5 cigarros por dia, e $102(5,9 \%)$ das tardias forneceram esta informação $\left(\chi^{2}=3,17 ; p=0,7\right)$. Quanto ao consumo de álcool, duas adolescentes precoces $(0,6 \%)$ e $12(0,7 \%)$ das tardias o faziam $(p=0,62)$. O uso de drogas ilícitas foi relatado por duas pacientes em cada grupo $(0,6 \%$ no grupo das adolescentes precoces e $0,1 \%$ no grupo das tardias; $p=0,11$ ).

$\mathrm{O}$ acesso ao pré-natal foi verificado na maioria das pacientes: $87 \%(280 / 322)$ das precoces e $88 \%(1.534 / 1.736)$ das tardias. No entanto, o número de consultas foi considerado insuficiente na maior parte dos casos: 63,9\% (179/280) das adolescentes precoces e 58,8\% (902/1.534) das tardias compareceram a menos de 6 consultas durante todo o acompanhamento pré-natal $\left(\chi^{2}=2,58 ; \mathrm{p}=0,1\right)$.

As principais intercorrências clínicas descritas foram: pré-eclâmpsia, infecção do trato urinário e anemia. A pré-eclâmpsia foi diagnosticada em $13,7 \%(44 / 322)$ das adolescentes precoces e $14,9 \%$ $(259 / 1.736)$ das tardias $\left(\chi^{2}=0,34 ; p=0,5\right)$. A razão de prevalência (RP) foi de 0,92 [IC 95\%:0,68-1,23]. A infecção do trato urinário foi encontrada em $6,5 \%(21 / 322)$ das adolescentes precoces e $6,3 \%$ $(110 / 1.736)$ das tardias $\left(\chi^{2}=0,01 ; p=0,9\right) ; R P=1,03$ [IC 95\%:0,66-1,62]. A anemia foi verificada em $14,9 \%(48 / 322)$ das adolescentes precoces e $12,6 \%$ $(219 / 1.736)$ das tardias $\left(\chi^{2}=1,2 ; p=0,2\right)$.

A maioria das pacientes $(68,7 \%)$ teve parto vaginal, sendo $65 \%$ parto natural e $3,7 \%$ fórcipe. A freqüência de cesariana no grupo das adolescentes precoces foi de $28,3 \%(91 / 322)$ e nas tardias, 
de $31,9 \%(554 / 1.736)\left(\chi^{2}=2,3 ; \mathrm{p}=0,3\right) ; \mathrm{RP}=0,85$ [IC 95\%= 0,68-1,05] (Tabela 1). Para o grupo das precoces, as principais indicações de cesárea, em ordem decrescente, foram: pré-eclâmpsia (22 casos: $24,2 \%$ ), distocia funcional (18 casos: $19,8 \%)$, desproporção céfalo-pélvica (18 casos: 19,8\%) e sofrimento fetal (12 casos: 13,2\%). No grupo das adolescentes tardias, a ordem foi: pré-eclâmpsia (150 casos: $27,1 \%$ ), cesárea de repetição (99 casos: $17,9 \%$ ), distocia funcional (97 casos: $17,5 \%$ ) e sofrimento fetal (63 casos: 11,4\%).

A idade gestacional no momento do parto variou de 26 a 42 semanas com média de 38,7 semanas. Houve $16,7 \%$ de partos prematuros. Um total de 65 partos no grupo das adolescentes precoces $(20,2 \%)$ e $279(16,1 \%)$ no grupo das tardias ocorreram com menos de 37 semanas de idade gestacional $\left(\chi^{2}=3,30 ; p=0,06\right) ; \mathrm{RP}=1,26$ [IC 95\% : $0,99-1,60]$.

Tabela 1 - Tipo de parto ocorrido nas gestantes adolescentes precoces (com menor de 16 anos) e tardias.

\begin{tabular}{lcccccc}
\hline \multirow{2}{*}{ Tipo de parto } & \multicolumn{2}{c}{ Cesárea* $^{*}$} & \multicolumn{2}{c}{ Fórcipe } & \multicolumn{2}{c}{ Natural } \\
\cline { 2 - 7 } & $\mathbf{n}$ & $\%$ & $\mathbf{n}$ & $\%$ & $\mathbf{n}$ & $\%$ \\
\hline Adolescentes precoces & 91 & 28,3 & 10 & 3,1 & 221 & 68,6 \\
Adolescentes tardias & 554 & 31,9 & 66 & 3,8 & 1.116 & 64,3 \\
Total & 645 & 31,3 & 76 & 3,7 & 1.337 & 65 \\
\hline
\end{tabular}

${ }^{*} X^{2}=2,30$ e $p=0,316$ NS RP $=0,85 ;$ [ [C 95\% : 0,68-1,05] NS.

Precoces: adolescentes com menos de 16 anos de idade; Tardias: adolescentes com 16 anos completos até 19 anos e 364 dias de idade. RP = razão de prevalência; IC 95\% = intervalo de confiança a $95 \%$.

Tabela 2 - Adequação do peso do recém-nascido conforme a idade gestacional no parto das gestantes adolescentes precoces (até 16 anos) e tardias.

\begin{tabular}{lcccccc}
\hline \multirow{2}{*}{ Peso RN x IG } & \multicolumn{2}{c}{ PIG $^{*}$} & \multicolumn{2}{c}{ AIG } & \multicolumn{2}{c}{ GIG } \\
\cline { 2 - 7 } & $\mathbf{n}$ & $\%$ & $\mathbf{n}$ & $\%$ & $\mathbf{n}$ & $\%$ \\
\hline Adolescentes precoces & 40 & 12,4 & 272 & 84,5 & 11 & 3,4 \\
Adolescentes tardias & 182 & 10,4 & 1.514 & 86,2 & 60 & 3,5 \\
Total & 222 & 10,7 & 1.786 & 85,9 & 71 & 3,4 \\
\hline
\end{tabular}

${ }^{*} X^{2}=1,17 ; p=0,55$ NS.

RN: recém-nascido; IG: idade gestacional no parto; PIG: recém-nascido pequeno para a idade gestacional; AIG:recém-nascido adequado para a idade gestacional; GIG: recém-nascido grande para a idade gestacional
Os recém-nascidos das adolescentes precoces apresentaram pesos variando de 725 a $4.430 \mathrm{~g}$ (média de $2.926 \pm 622 \mathrm{~g}$ ). O peso médio dos RN das adolescentes tardias foi de $3.002 \mathrm{~g} \mathrm{(} \pm 656 \mathrm{~g}$ ) variando de 545 a $4.610 \mathrm{~g}$. A freqüência de RN pequenos para a idade gestacional foi de $10,8 \%(222 / 2.058)$. No grupo das gestantes precoces 12,4\% (40/322) eram PIG e no grupo das tardias 10,5\% (182/1.736) apresentavam esta complicação $\left(\chi^{2}=1,17 ; \mathrm{p}=0,55\right)$ (Tabela 2).

Houve 308 (15,1\%) RN com indice de Apgar no primeiro minuto menor que 7 , sendo 64 (19,9\%) no grupo das adolescentes precoces e 244 (14,2\%) das tardias $\left(\chi^{2}=6,96 ; p=0,008\right) ; R P=1,4$ [IC 95\% : $1,10-1,80]$. No quinto minuto, 71 (3,6\%) dos RN ainda encontravam-se com Apgar menor que 7, sendo que $17(5,3 \%)$ pertenciam ao grupo das precoces e $54(3,3 \%)$ ao grupo das tardias $\left(\chi^{2}=3,01\right.$; $\mathrm{p}:$ 0,08); RP=1,6 [IC 95\% : 0,94-2,72] (Tabela 3).

Malformações congênitas foram diagnosticadas em $10(3,1 \%)$ RN no grupo das adolescentes precoces e $46(2,7 \%)$ dos RN das tardias $\left(\chi^{2}=0,198\right.$; $\mathrm{p}=0,656)$. Os defeitos faciais foram os mais freqüentes entre as precoces ( 4 casos: $1,3 \%$ ), seguidos das alterações músculo-esqueléticas (0,9\%). Entre os RN das adolescentes tardias o defeito congênito mais freqüente foi cardiopatia, com 13 casos $(0,8 \%)$, seguido dos defeitos do tubo neural $(0,6 \%)$.

Houve 58 (2,8\%) óbitos neonatais. Destes, 5 casos $(1,6 \%)$ ocorreram nos filhos de adolescentes precoces e $53(3,1 \%)$ nos filhos das adolescentes tardias $\left(\chi^{2}=2,25 ; \mathrm{p}=0,133\right) ; \mathrm{RP}=0,51$ [IC 95\% : $0,20-1,26]$.

\section{Discussão}

Vários estudos indicam que a gravidez nas adolescentes se associa com aumento dos riscos gestacionais ${ }^{2,9,10}$. Os mecanismos biológicos responsáveis por essa diferença permanecem especulativos. Já foi atribuída ao fato de que mulheres muito jovens continuam a crescer durante a gravidez e podem competir com o feto por nutrientes ${ }^{2}$.

Tabela 3 - Índices de Apgar registrados no primeiro e quinto minuto de vida nos recém-nascidos das gestantes adolescentes precoces (até 16 anos de idade) e tardias.

\begin{tabular}{|c|c|c|c|c|c|c|c|c|}
\hline & \multicolumn{4}{|c|}{ Apgar 1 minuto* } & \multicolumn{4}{|c|}{ Apgar 5 minutos ${ }^{* *}$} \\
\hline & \multicolumn{2}{|c|}{$<7$} & \multicolumn{2}{|c|}{$\geq 7$} & \multicolumn{2}{|c|}{$<7$} & \multicolumn{2}{|c|}{$\geq 7$} \\
\hline & $\mathrm{n}$ & $\%$ & $\mathrm{n}$ & $\%$ & $n$ & $\%$ & $\mathrm{n}$ & $\%$ \\
\hline Adolescentes precoces & 64 & 19,9 & 257 & 80,1 & 17 & 5,3 & 305 & 94,7 \\
\hline Adolescentes tardias & 244 & 14,2 & 1.475 & 85,8 & 54 & 3,3 & 1.581 & 96,7 \\
\hline Total & 308 & 15,1 & 1732 & 84,9 & 71 & 3,6 & 1.886 & 96,4 \\
\hline
\end{tabular}

$\mathrm{RP}=$ razão de prevalência; IC $95 \%$ = intervalo de confiança a $95 \%$. 
Os resultados obstétricos em adolescentes continuam incertos, bem como persiste a incerteza se adolescentes mais jovens teriam prognóstico diferente daquelas de mais idade. Em nosso estudo nós comparamos duas subpopulações de adolescentes (precoces e tardias) e não encontramos diferenças significativas quanto ao risco gestacional. Esses resultados diferem de outros estudos realizados também em países em desenvolvimento ${ }^{1,11}$.

Em estudo com adolescentes da América Latina, foram analisadas 344.626 gestações. A população de estudo foi dividida em 3 grupos: com menos de 15 anos, 16 a 17 anos e 18 a 19 anos. Observouse clara tendência para freqüência aumentada de pré-eclâmpsia, eclâmpsia, anemia e cesariana com a diminuição da idade materna. No primeiro grupo a freqüência de pré-eclâmpsia foi de 5,9\% e de anemia de $8,8 \%$. No terceiro grupo os valores encontrados foram, 4,3 e 6,2\%, respectivamente. A freqüência de infecção do trato urinário foi de $4,3 \%$ para todos os grupos de adolescentes. Estes valores são inferiores aos encontrados no nosso estudo, que revelou: préeclâmpsia em 13,7\% das adolescentes precoces e $14,9 \%$ das tardias e anemia em $14,9 \%$ das pacientes mais jovens e $12,6 \%$ nas de mais idade ${ }^{1}$.

Para alguns autores, as cifras apresentadas para pré-eclâmpsia revelam-se mais elevadas entre adolescentes, principalmente nas de menor idade ${ }^{1}$. A toxemia seria particularmente mais incidente nas adolescentes mais jovens (menor de 16 anos) porque neste grupo é maior o número de pacientes nulíparas, com estado nutricional deficiente e ausência de cuidado pré-natal. Nossos dados revelam que não houve diferença entre as duas faixas etárias. A falta de critérios precisos para o diagnóstico dessa complicação no decorrer dos anos talvez possa explicar essa diferença nos achados.

A anemia é de grande relevância na área obstétrica, dada sua ampla ocorrência, (entre 20 a $80 \%$ nas diversas populações), bem como por suas repercussões tanto para o lado materno como fetal $^{12,13}$. Das nossas adolescentes $12,9 \%$ eram anêmicas, não havendo diferença significante entre as duas faixas etárias.

Ao analisar as gestações em adolescentes de Minas Gerais, também se encontraram resultados perinatais piores na população com idade de 10 a 14 anos de idade quando comparada àquelas com 15 a 19 anos, exceto pela incidência de cesariana, que foi semelhante (20 vs $21 \%$ ). A taxa de prematuridade $(25,5$ vs $7,4 \%)$ e a freqüência de baixos escores de Apgar no quinto minuto $(6,06$ vs 3,3\%) e de baixo peso ao nascer $(35,2$ vs $11,7 \%)$ foram mais altas na população de menor idade. Após ajuste em relação ao número de consultas pré-natais, essa diferença se manteve ${ }^{11}$.
Foi sugerido que, em adolescentes jovens, os ossos pélvicos e o canal de parto podem ainda estar em processo de crescimento, aumentandose a indicação de cesárea por parto obstruído ou prolongado. No entanto, contrariando essa teoria da imaturidade biológica, a freqüência de cesárea em muitas casuísticas com adolescentes tem sido menor que em mulheres adultas ${ }^{1,9,10,14,15}$. As razões para esta associação não são claras. Uma possivel explicação seria a significativamente alta incidência de baixo peso ao nascer em adolescentes ${ }^{2,14,16,17}$, que poderia estar associada com maior chance de parto via vaginal e a menor incidência de cesárea de repetição, tão freqüente em adultas.

A incidência de cesárea no nosso estudo foi bastante elevada $(28,3 \%$ nas adolescentes precoces e $31,9 \%$ nas tardias), freqüência esta superior à encontrada por outros autores ${ }^{1,11}$. Em nosso estudo, não avaliamos a incidência de baixo peso ao nascer; no entanto, a incidência de prematuridade (16,7\% da população total) e de RN pequenos para a idade gestacional $(10,7 \%)$ foram relativamente elevadas, contribuindo sobremaneira com muitos RN de baixo peso.

Quando se comparam diferentes faixas de idade na adolescência há tendência de achar freqüência inferior de cesariana em grupos de maior idade $^{1}$. Nosso estudo revelou a mesma freqüência de cesariana e parto a fórcipe nas adolescentes tardias e precoces.

Os resultados perinatais foram diferenciados de estudo com adolescentes na América Latina ${ }^{1}$. Foi encontrada maior freqüência de RN pequenos para a idade gestacional, partos pré-termos e morte neonatal precoce no grupo de menor idade. Os baixos indices de Apgar no quinto minuto foram igualmente freqüentes em todos os grupos. Diferentemente dos nossos resultados esses autores concluíram que as adolescentes, principalmente aquelas mais jovens que 16 anos no parto, possuem aumento de risco para vários resultados obstétricos adversos ${ }^{1}$. Observação semelhante também foi encontrada por outros autores que avaliaram adolescentes de países em desenvolvimento ${ }^{7,9}$.

Com o objetivo de definir qual o limite de idade para que a adolescente fosse considerada de risco, alguns autores estabeleceram que no grupo com menos de 16 anos houve maior freqüência de RN com muito baixo peso, pré-termos e mortalidade infantil que nas adolescentes com 16 anos ou mais. Portanto, consideraram ser este o limite de divisão entre dois grupos na adolescência. No entanto, encontraram que as características são diferentes entre as duas populações. As pacientes com menor idade apresentavam tendência à assistência pré-natal inadequada e ganho de peso 
deficiente durante a gravidez, mas em compensação havia menor freqüência do uso de fumo ou álcool ${ }^{7}$. Não se avaliou, no nosso estudo, ganho de peso durante a gestação, mas no que diz respeito à assistência pré-natal e uso de fumo ou drogas ilícitas nossos dois grupos eram semelhantes, podendo, inclusive, justificar os achados diferentes dos encontrados por estes autores.

Há controvérsias na literatura sobre os fatores responsáveis pela maior freqüência de resultados obstétricos adversos em adolescentes. Fatores socioeconômicos, tais como assistência pré-natal inadequada, pobreza, baixo nível educacional, gravidez não desejada, estresse psicológico e uso de drogas ilícitas, são geralmente apontados como importantes determinantes dos piores índices de complicações nestas pacientes ${ }^{1,15,18}$. Comparamos nossos dois grupos quanto à assistência pré-natal (acesso e número de consultas), uso de fumo e drogas ilícitas e eles se mostraram estatisticamente semelhantes, eliminando, portanto, esses importantes fatores complicadores na comparação entre as duas populações. Toda nossa casuística pertencia a classes sociais menos favorecidas. Mais adolescentes tardias eram multiparas, se declararam casadas e com maior tempo de estudo, parâmetros que podem refletir exatamente o maior tempo de vida do grupo e não apenas diferenças estatísticas entre eles. Consideraram-se, portanto, os dois grupos semelhantes do ponto de vista de outros riscos gestacionais que não apenas a idade materna, já que o objetivo principal do estudo foi comparar duas populações de adolescentes (precoces e tardias) e seus resultados obstétricos.

Observamos que os dois grupos não apresentaram resultados obstétricos diferentes. Não houve diferença quanto à ocorrência de pré-eclâmpsia, anemia ou ITU, freqüência de cesárea ou parto a fórcipe, prematuridade, baixo índice de Apgar de quinto minuto, $\mathrm{RN}$ pequeno para a idade gestacional, anomalias congênitas ou morte neonatal. $O$ único critério avaliado com diferença estatisticamente significativa foi a presença de baixos escores de Apgar de primeiro minuto, que foi mais comum nas adolescentes precoces $(19,9$ vs 14,2\%). Mais estudos são necessários com exclusão de outros fatores que possam interferir na análise, como, por exemplo, multiparidade, ganho de peso no pré-natal, raça, nivel de escolaridade e presença de união marital estável.

Conclui-se que gestantes adolescentes precoces e tardias, submetidas às mesmas condições socioeconômicas e de assistência pré-natal, apresentam resultados semelhantes em relação à evolução da gestação, tendo ambas características biológicas aceitáveis para desempenho obstétrico satisfatório.

\section{Referências}

1. Conde-Agudelo A, Belizan JM, Lammers C. Maternalperinatal morbidity and mortality associated with adolescent pregnancy in Latin America: cross-sectional study. Am J Obstet Gynecol. 2005;192(2):342-9.

2. Fraser AM, Brockert JE, Ward RH. Association of young maternal age with adverse reproductive outcomes. N Engl J Med. 1995;332(17):1113-7.

3. Goldenberg RL, Klerman LV. Adolescent pregnancy: another look. N Engl J Med. 1995;332(17):1161-2.

4. Mahfouz AA, el-Said MM, al-Erian RA, Hamid AM. Teenage pregnancy: are teenagers a high risk group? Eur J Obstet Gynecol Reprod Biol. 1995;59(1):17-20.

5. Al-Ramahi M, Saleh S. Outcome of adolescent pregnancy at a university hospital in Jordan. Arch Gynecol Obstet. 2006;273(4):207-10.

6. Scholl TO, Hediger ML, Belsky DH. Prenatal care and maternal health during adolescent pregnancy: a review and meta-analysis. J Adolesc Health. 1994;15(6):444-56.

7. Phipps MG, Sowers M. Defining early adolescent childbearing. Am J Public Health. 2002;92(1):125-8.

8. Huffman JW. Childhood and adolescent pregnacies. In: Huffman JW, editor. The gynecology of childhood and adolescence. Philadelphia: WB. Saunders; 1981. p. 560-9.

9. Jolly MC, Sebire N, Harris J, Robinson S, Regan L. Obstetric risks of pregnancy in women less than 18 years old. Obstet Gynecol. 2000;96(6):962-6.

10.Satin AJ, Leveno KJ, Sherman ML, Reedy NJ, Lowe TW, McIntire DD. Maternal youth and pregnancy outcomes: middle school versus high school age groups compared with women beyond the teen years. Am J Obstet Gynecol. 1994;171(1):184-7.

11.Goldenberg P, Figueiredo MCT, Silva RS. Gravidez na adolescência, pré-natal e resultados perinatais em Montes Claros, Minas Gerais, Brasil. Cad Saúde Pública. 2005;21(4):1077-86.

12.Papa ACE, Furlan JP, Pasquelle M, Guazzelli CAF, Figueiredo MS, Camano L, et al. A anemia por deficiência de ferro na grávida adolescente: comparação entre métodos laboratoriais. Rev Bras Ginecol Obstet. 2003;25(10):731-8.

13. Escobar A, Bálcazar N. Factores de riesgo y características de la gestación en la adolescencia y su hijo. Rev Colomb Obstet Ginecol. 1995;46(2):109-16.

14.Ziadeh S. Obstetric outcome of teenage pregnancies in North Jordan. Arch Gynecol Obstet. 2001;265(1): 26-9.

15.Lubarsky SL, Schiff E, Friedman SA, Mercer BM, Sibai BM. Obstetric characteristics among nulliparas under age 15. Obstet Gynecol. 1994;84(3):365-8. 
16. Pavlova-Greenfield TP, Sutija VG, Gudavalli M. Adolescent pregnancy: positive perinatal outcome at a community hospital. J Perinat Med. 2000;28(6):443-6.

17.Yildirim Y, Inal MM, Tinar S. Reproductive and obstetric characteristics of adolescent pregnan- cies in Turkish women. J Pediatr Adolesc Gynecol. 2005;18(4):249-53.

18. Figueiredo B, Pacheco A, Magarinho R. Grávidas adolescentes e grávidas adultas: diferentes circunstâncias de risco? Acta Med Port. 2005;18(2):97-105. 